\title{
UAV UWB POSITIONING CLOSE TO BUILDING FACADES: A CASE STUDY
}

\author{
M. P. Bouloukou ${ }^{1}$, A. Masiero ${ }^{2 *}$ A. Vettore $^{3}$, V. Gikas ${ }^{1}$ \\ ${ }^{1}$ School of Rural and Surveying Engineering, National Technical University of Athens (NTUA), \\ Athens, Greece - vgikas@central.ntua.gr \\ ${ }^{2}$ Dept. of Civil and Environmental Engineering, University of Florence, \\ via di Santa Marta 3, Florence 50139, Italy - andrea.masiero@unifi.it \\ ${ }^{3}$ Interdepartmental Research Center of Geomatics (CIRGEO), University of Padova, \\ Viale dell'Università 16, Legnaro (PD) 35020, Italy - antonio.vettore@ unipd.it
}

\section{Commission I, WG I/7}

KEY WORDS: UAV, UWB, Positioning, EKF, GNSS-denied environments.

\begin{abstract}
:
Nowadays, Unmanned Aerial Vehicles represent a very popular tool used in dramatically wide range of applications: indeed, their high flexibility, ease of use, and in certain cases quite affordable price make them a very attractive solutions in a number of applications, including surveying and mapping. Despite such a wide range of uses, their usage in automatic/autonomous mode is still restricted by the requirement of the availability of a reliable positioning and navigation system, which in practically all the commercial solutions is represented by the Global Navigation Satellite System (GNSS). Unfortunately, the availability and reliability of GNSS cannot be ensured in all the working conditions of interest. In particular, such condition may not hold downtown, close to high buildings. Since this can also be an operative condition of wide interest, this paper aims at investigating the use of an alternative positioning method that can be integrated with GNSS in order to compensate its unavailability. To be more specific, this paper investigates the positioning performance of an Ultra Wide-Band (UWB) system when an UWB rover is attached to a drone flying close to a building facade, whereas a set of UWB anchors are on the ground, close to the facade. The results obtained in the case study of a building of the University of Padua show that the UWB system positioning performance is quite good (quite less than 1 meter error for most of the time) up to approximately 15-20 meters of distance from the anchors. Close to the top of the building the error significantly increases when using an Extended Kalman filter (EKF) positioning approach, probably mostly due to the low UWB measurement success rate at such heights and to the poor geometric configuration of the UWB network. Nevertheless, a Gauss-Newton-based positioning strategy outperforms the EKF in such critical case, still ensuring errors at 1 meter level.
\end{abstract}

\section{INTRODUCTION}

The popularity of Unmanned Aerial Vehicles (UAVs) has dramatically grown during the last decade, and, nowadays, their use is common in a large number of applications in several fields, such as civil engineering, building modeling and monitoring (Piras et al., 2017), environmental monitoring, forestry (Casbeer et al., 2006), precision agriculture (Zhang and Kovacs, 2012), disaster management and civil security (Maza et al., 2011, Baiocchi et al., 2013), and mapping (Remondino et al., 2011, Lo et al., 2015, Pepe et al., 2018), just to cite some. Readers are referred for instance to (Pajares, 2015, Colomina and Molina, 2014) for more complete overviews on the UAV applications.

Given their commonly accepted utility in such a wide range of applications, the research community has recently focused on extending the usability of UAVs in more general scenarios, in particular to compensate the absence or unreliability of Global Navigation Satellite System (GNSS). Indeed, currently UAV navigation is enabled by the presence of a GNSS positioning system, which ensures the movements of the UAV to the correct locations. However, it is well known that GNSS positioning is not available, or not reliable, in a certain number of working conditions, e.g. indoors, in urban canyons. Consequently, some efforts have been made to develop positioning approaches able

\footnotetext{
* Corresponding author
}

to substitute GNSS in such cases. Among the proposed solutions to such aim, some works, similarly to this paper, have recently considered the use of Ultra Wide-Band devices for indoors (Tiemann et al., 2015) and other environments challenging for GNSS, in certain cases integrating UWB measurements with information provided by other sensors (Zahran et al., 2018).

Similarly to such approaches, this paper focuses on the use of an UWB positioning system in order to properly track drone movements close a to a building façade. This case study is of particular interest for instance when aiming at using a UAV to monitoring the health status of a building façade.

An UWB rover attached to the UAV acquires range measurements by communicating with a set of UWB anchors distributed on the ground, close to the building façade.

Two different strategies are used to properly process such UWB ranges: first, an Extended Kalman filter (EKF) approach is implemented to track the UAV positioning.

Then, an iterative Gauss-Newton approach is implemented to directly iteratively solve the trilateration problem associated to the computation of the UAV position, based on the UWB range measurements between the rover and the anchors.

More details about the case study and the positioning methods are provided in Section 2 and 3, respectively, whereas the ob- 
tained positioning results are shown in Section 4, and, finally, some discussion and conclusions are drawn in Section 5 and 6.

\section{CASE STUDY}

This work investigates the use of an affordable UWB positioning system, namely Pozyx (Pozyx Labs, 2015), to assess the position of an UAV while the latter is performing a monitoring task on a building façade. In order to accomplish such monitoring goal, the drone flies very close to the façade $(\approx 1 \mathrm{~m})$, and it occasionally lingers on certain locations, if needed.

During the data collection related to the considered case study, monitoring a building of the University of Padua, an UWB rover was mounted on the UAV, and eight static UWB anchors were positioning on the ground, just a few meters far from the building façade. UWB rover and anchors communicated during all the test, collecting a set of range measurements.

Fig. 1 shows the drone used for the data collection, i.e. a DJI Phantom 4 Pro, and the external sensors attached to it: a Pozyx rover and an external GNSS receiver, Emlid Reach M+ (Emlid Ltd, 2019), working in post processing kinematic (permanent station was at less than 100 meters from the case study building).

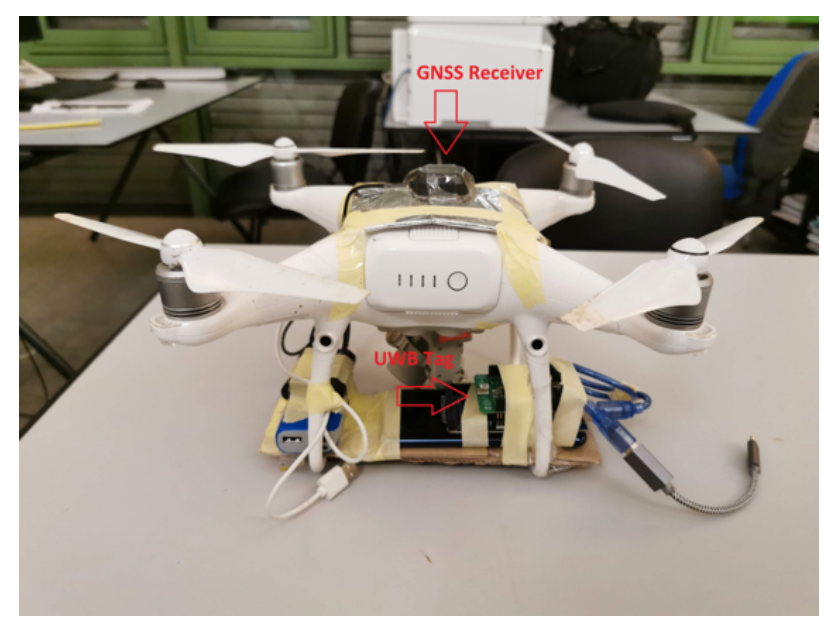

Figure 1. External sensors attached to the drone.

Fig. 2 shows certain of the eight Pozyx UWB anchors on the ground, close to the building façade, whereas Fig. 3 shows the drone during the data acquisition, i.e. while flying close to the building for monitoring purposes.

During all the flight, the UWB rover iteratively checked the availability of new range measurements from each of the anchors in a loop. Eight is clearly the maximum amount of range measurements that could be collected in a loop, however, the rover rarely gathered more than five ranges per loop, as shown in Figure 4.

Despite some other works already investigated the use of UWB devices for UAV positioning, this work has the peculiarity of testing the performance of this approach in the specific case of flying very close to a building wall. Differently from other working scenarios (Gabela et al., 2019), where the same devices have shown quite good performance in terms of accuracy and maximum range, in this case study the percentage of successful range measurements is much lower (see again Figure 4). A

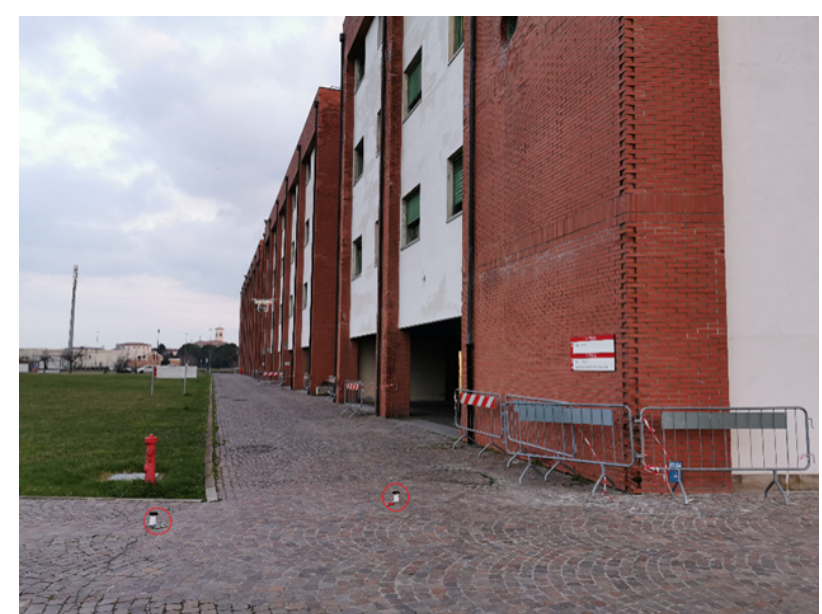

Figure 2. Pozyx UWB anchors distributed on the ground close to the building façade.

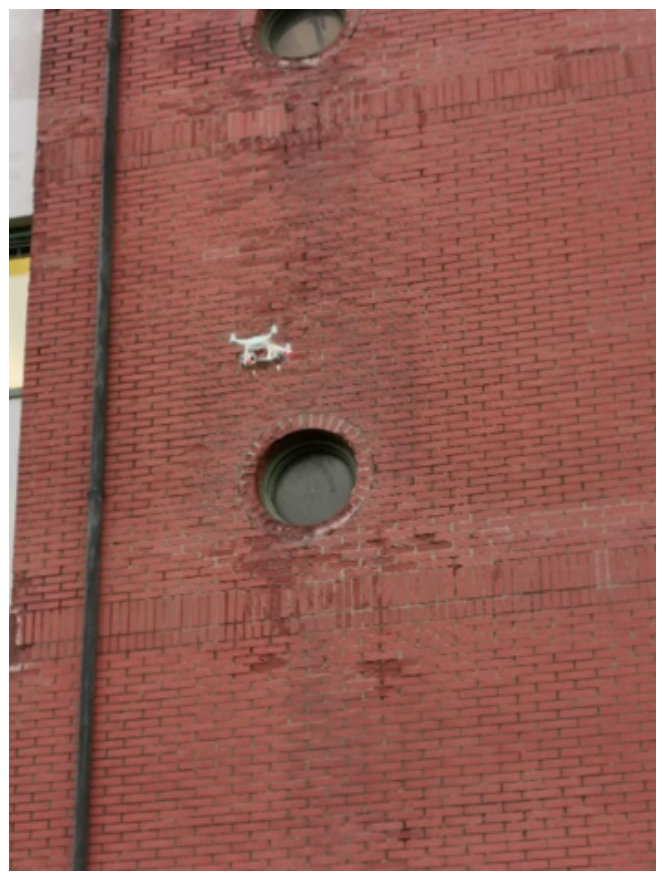

Figure 3. Drone flying close to the building façade.

possible explanation might be the influence of the thick building façade on the UWB measurements. Furthermore, since in this case study all the anchors were on the ground, the geometric UWB network configuration was particularly poor considering the goal of assessing the UAV position (Dabove et al., 2018), in particular when the UAV was quite far from the ground.

Such UWB measurements have been combined by means of an Extended Kalman filter (EKF) (Masiero et al., 2019), which allowed to quite effectively track the UAV movements during the data collection. The tracking results obtained by means of the EKF are also compared in the following section with those obtained by repetitively computing the UAV positions by directly solving a trilateration problem by means of a Gauss-Newton algorithm.

Furthermore, the case study has been selected in such a way to ensure that GNSS positioning was effectively working for a significant part of the test duration (e.g. the closest buildings 


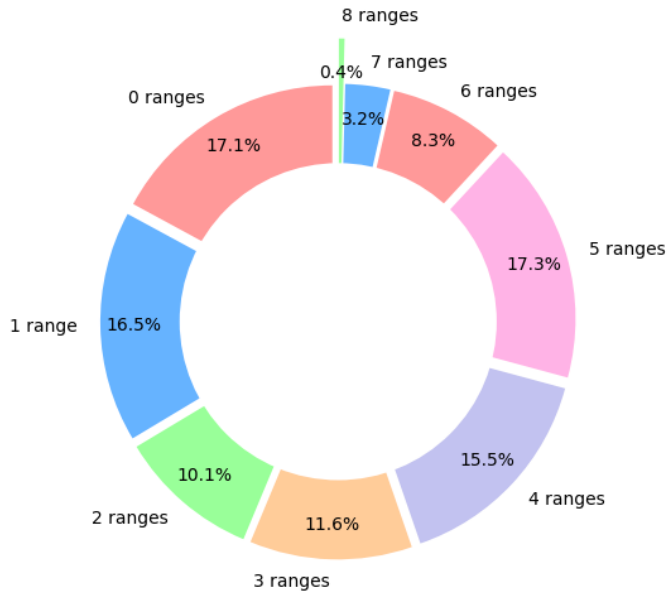

Figure 4. Number of available ranges in a loop (percentage of the cases)

and trees were far from the considering façade, hence reducing the potential issues on GNSS positioning in a urban environment). In particular, GNSS measurements were post-processed exploiting the corrections provided by a permanent station very close to the considered façade $(\approx 100 \mathrm{~m}$ far). Hence, GNSS measurements, in fixed working conditions, are used in this work as a reference trajectory, to validate UWB estimates.

\section{POSITIONING STRATEGIES}

Two kinds of positioning strategies have been considered in this work. Both of them aims at properly processing the UWB ranges, despite in a slightly different way:

- First, an Extended Kalman filter (EKF) has been employed: let the state vector $\mathbf{x}_{k}$ at time $t_{k}$ be defined as the rover position and velocity: $\mathbf{x}_{k}=\left[\mathbf{p}\left(t_{k}\right)^{\top} \mathbf{v}\left(t_{k}\right)^{\top}\right]^{\top}$.

Then, the model $\mathbf{x}_{k+1}=F_{k} \mathbf{x}_{k}+\omega_{k}$, where $\omega_{k}$ is a Gaussian distributed zero-mean white noise process, is used to describe the dynamic of the drone from time $t_{k}$ to $t_{k+1}$, where $t_{k+1}-t_{k}=\Delta t_{k+1}$. The dynamic matrix $F_{k}$ is defined as follows:

$$
F_{k}=\left[\begin{array}{cc}
I & \Delta t_{k} I \\
0 & I
\end{array}\right]
$$

The measurement equation is instead:

$$
z_{k}^{j}=\left|\mathbf{p}\left(t_{k}\right)-\mathbf{p}^{j}\right|+\xi_{k}
$$

where $\xi_{k}$ is assumed to be a Gaussian distributed zeromean white noise process, whereas $\mathbf{p}^{j}$ is the position of the $j$-th UWB anchor.

- Differently, the second proposed approach directly computes the drone position by solving the trilateration problem given the measured UWB ranges. The Gauss-Newton algorithm is used to compute the solution of such positioning problem at each iteration of the rover positioning algorithm. At each iteration, the Gauss-Newton takes advantage of the solution computed at the previous time instant by using it as an initial solution of the trilateration problem.
The reader is referred for instance to (Schaback, 1985) for more details about the Gauss-Newton optimization algorithm.

\section{RESULTS}

First, Figure 5 shows a comparison of the range measurements between the UWB rover and one of the anchors compared with the corresponding distances computed by means of the GNSS.

Then, Figure 6 summarizes the ranging success rate of the UWB system as a function of the distance between anchors and rover. It is quite apparent the significant decrease of the success rate for ranges larger than not so much more than 10 meters.

Nevertheless, it is worth to notice that this working condition seems to be quite peculiar of the scenario considered in this work: indeed, in different working conditions the same UWB system can successively perform the ranging at much larger distances.

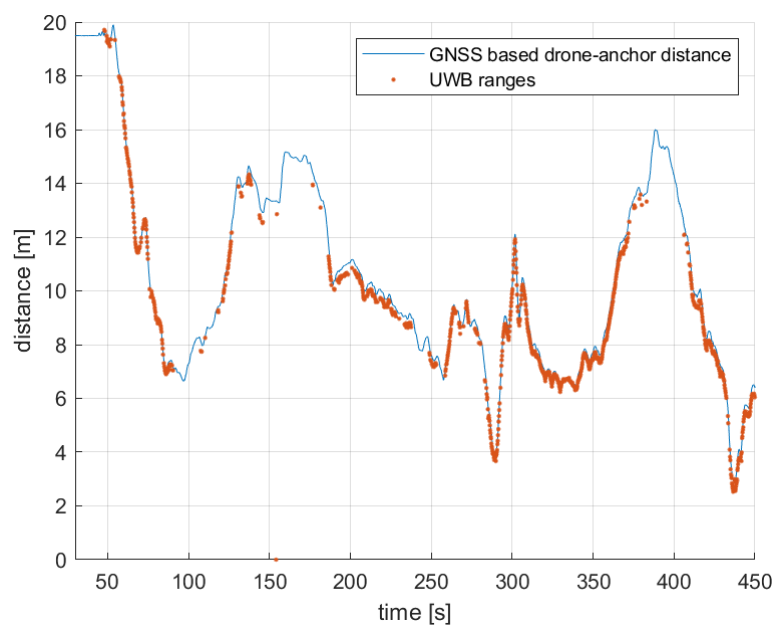

Figure 5. Example of range measurements between the drone and an anchor positioned on the ground (red dot marks), compared with GNSS-based ground truth distance (blue solid line).

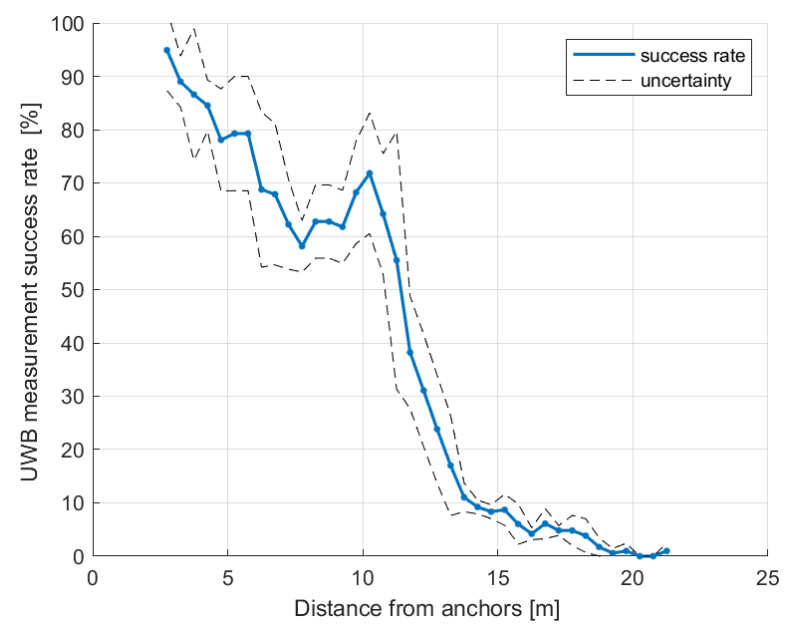

Figure 6. UWB measurement success rate varying the drone distance with respect to the anchors.

Figure 7 shows the 3D positioning error of the UWB estimates, 
obtained by means of the EKF, with respect to the GNSS reference trajectory.

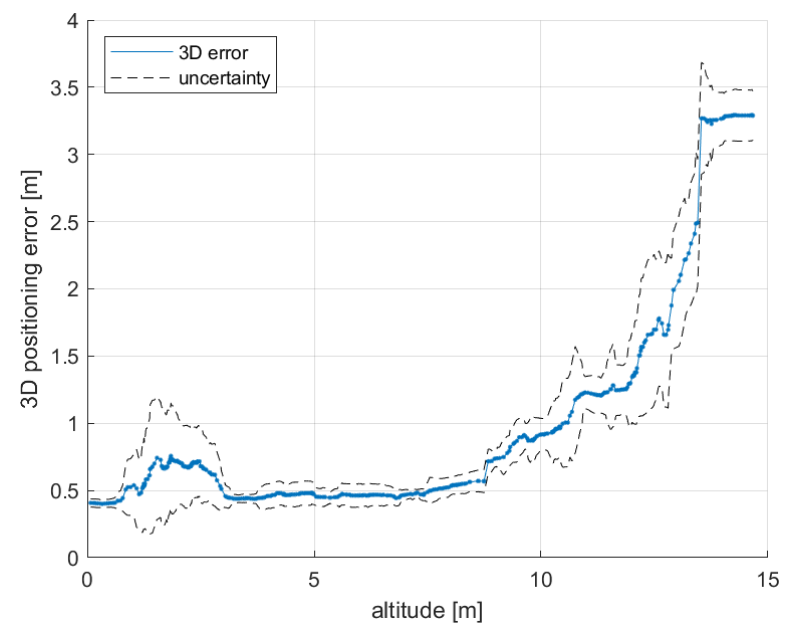

Figure 7. 3D positioning error varying the drone altitude.

The error in Figure 7 increases significantly after 10 meters of altitude. Such increase is mostly determined by large height errors, as show in Figure 8.

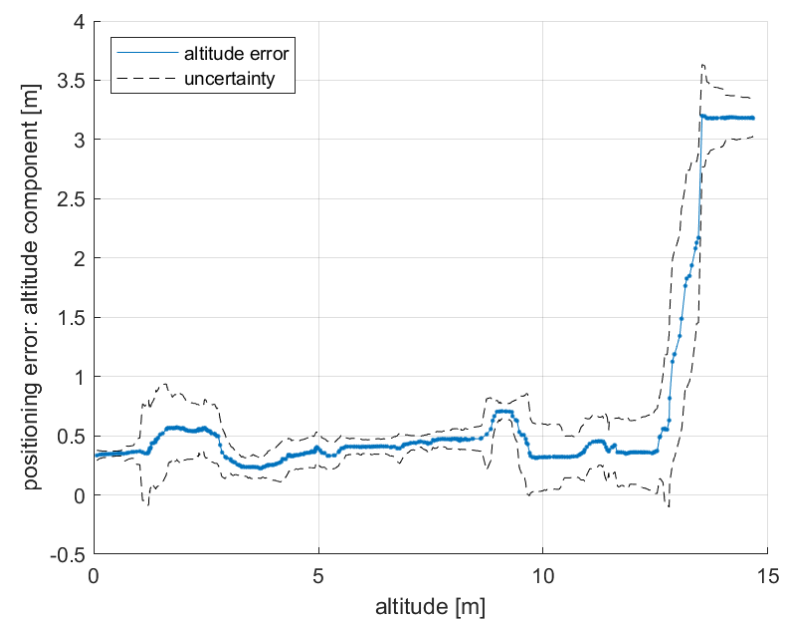

Figure 8. Height component of the positioning error varying the drone altitude.

For what concerns the Gauss-Newton-based positioning approach, Figure 9 and 10 show the obtained 3D positioning error and the height component of such error, respectively, as functions of the flying altitude.

\section{DISCUSSION}

The results shown in the previous section show that the EKF approach seems to be quite effective to determine the UAV positioning up to a certain altitude, e.g. in this case study the 3D positioning error is less or equal to 1 meter up to ten meter of altitude (see Figure 7), approximately. Then, the height component of the positioning error becomes quite large (Figure 8).

A first explanation for this kind of behavior of the EKF 3D positioning error is the low availability of measurements close to the top of the building. Indeed, as shown in Figure 6, in this case study the UWB measurement success rate decreases dramatically for distances larger than 10 meters from the anchors.

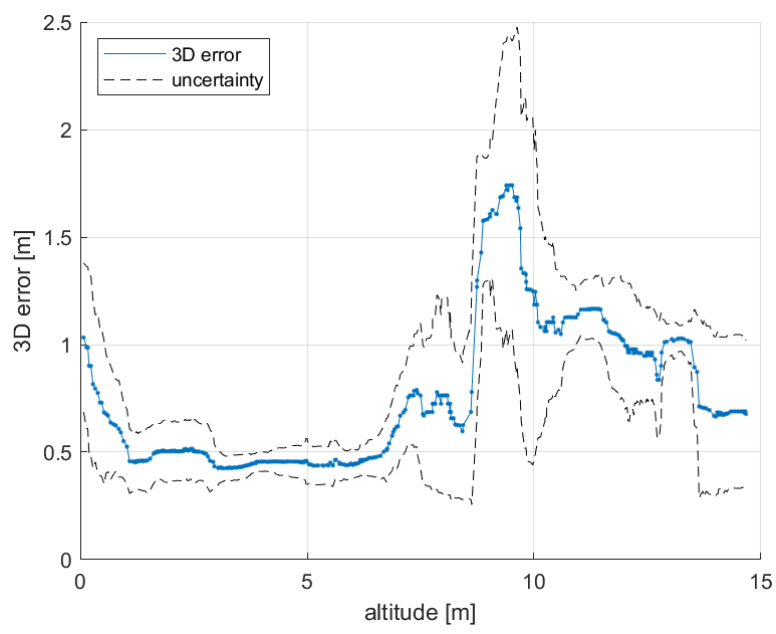

Figure 9. 3D positioning error varying the drone altitude (Gauss-Newton algorithm based estimates).

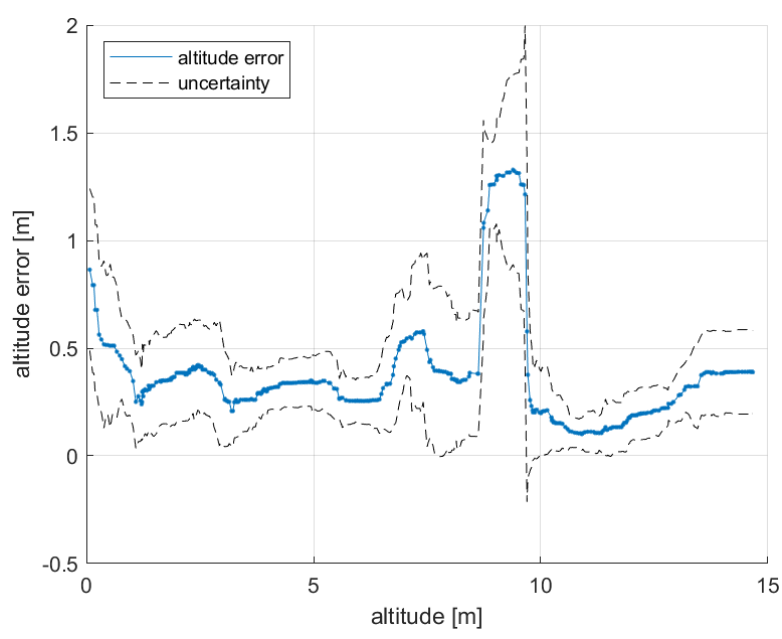

Figure 10. Height component of the positioning error varying the drone altitude (Gauss-Newton algorithm based estimates).

Since such decrease in the measurement success rate is quite strange outdoors, it may be due to the closeness of the drone to the building.

Despite this can be a limitation in real applications, it is worth to notice that it can be at least partially reduced by attaching the anchors directly on the building walls (e.g. on the windows). Despite not implemented in this work, such option may make the performance of the system much more effective in a real scenario, in particular when using the drone close to quite high buildings.

Furthermore, the above potential solution shall also have positive drawbacks on the geometric configuration of the UWB network: indeed, the configuration considered in this work, based just on a set of eight anchors positioned on the ground, is clearly characterized by a quite poor geometric robustness. The presence of additional anchors distributed on the building walls shall quite improve the overall robustness of the UWB geometric configuration, potentially resulting in an improvement also on the positioning results.

Despite the UWB network geometric configuration is surely quite poor in the case study considered in this work, a possible 
motivation for the poor performance of the EKF close to the top of the building shall also be related to the algorithm itself.

Indeed, the second proposed approach, based on the iterative use of the Gauss-Newton optimization algorithm in order to properly solve the trilateration problem, and hence compute the UAV position, effectively tracks the UAV even close to the top of the building.

Despite even in this case the 3D positioning error tends to increase for larger altitudes, its peak in the considered dataset is at approximately 10 meters of altitude, whereas then it slightly decreases, to values not that different from those at low heights (see Figure 9).

This is also confirmed by Figure 10, where, differently from the EKF case, the height component of the positioning error has a peak around ten meters of altitudes, but, in general, it does not seem to be so much dependent on the flying height.

Overall, the Gauss-Newton approach is apparently more effective than the EKF for altitudes larger than 10 meters, whereas for lower altitudes their performance is quite similar.

\section{CONCLUSIONS}

This paper showed the performance of a positioning system based on UWB transceivers for tracking UAV movements close to a building façade, in order to compensate the potential unavailability of unreliability of GNSS.

In particular, it has been shown that the measurement success rate is largely dependent on the distance between anchors and rover, and, in this case study, it decreased dramatically fast for distances larger than 10-15 meters.

For what concerns the positioning performance of the proposed system: the results obtained by means of the Gauss-Newton approach apparently surpasses those of the EKF, showing a more stable performance at almost all the considered flying altitudes.

Our future works will consider further investigations on the robustness of the UWB network configuration, in particular focusing on the potential positioning performance improvement obtained by attaching UWB anchors also to the building walls.

Furthermore, some effort will also be dedicated to the development of cooperative UAV strategies, hence exploiting the UWB system also to ensure good estimates of the distance between any two drones (such method shall be more effective than other approaches also based on radio signals (Masiero et al., 2015)).

\section{REFERENCES}

Baiocchi, V., Dominici, D., Mormile, M., 2013. UAV application in post-seismic environment. ISPRS - International Archives of the Photogrammetry, Remote Sensing and Spatial Information Sciences, 1, W2.

Casbeer, D. W., Kingston, D. B., Beard, R. W., McLain, T. W., 2006. Cooperative forest fire surveillance using a team of small unmanned air vehicles. International Journal of Systems Science, 37(6), 351-360.

Colomina, I., Molina, P., 2014. Unmanned aerial systems for photogrammetry and remote sensing: A review. ISPRS Journal of Photogrammetry and Remote Sensing, 92, 79 - 97.
Dabove, P., Di Pietra, V., Piras, M., Jabbar, A. A., Kazim, S. A., 2018. Indoor positioning using ultra-wide band (uwb) technologies: Positioning accuracies and sensors' performances. 2018 IEEE/ION Position, Location and Navigation Symposium (PLANS), IEEE, 175-184.

Emlid Ltd, 2019. Emlid docs. https://emlid.com/.

Gabela, J., Retscher, G., Goel, S., Perakis, H., Masiero, A., Toth, C., Gikas, V., Kealy, A., Koppányi, Z., Błaszczak-Bak, W. et al., 2019. Experimental evaluation of a UWB-based cooperative positioning system for pedestrians in GNSS-denied environment. Sensors, 19(23), 5274.

Lo, C., Tsai, M., Chiang, K., Chu, C., Tsai, G., Cheng, C., El-Sheimy, N., Habib, A., 2015. The Direct Georeferencing Application and Performance Analysis of UAV Helicopter in GCP-Free Area. ISPRS - International Archives of Photogrammetry, Remote Sensing and Spatial Information Sciences, 40(1), 151.

Masiero, A., Fissore, F., Guarnieri, A., Pirotti, F., Vettore, A., 2015. UAV Positioning and Collision Avoidance Based on RSS Measurements. ISPRS - International Archives of Photogrammetry, Remote Sensing and Spatial Information Sciences, 40(1), 219.

Masiero, A., Fissore, F., Guarnieri, A., Pirotti, F., Vettore, A., 2019. Aiding indoor photogrammetry with UWB sensors. Photogrammetric Engineering \& Remote Sensing, 85(5), 369-378.

Maza, I., Caballero, F., Capitán, J., Martínez-de Dios, J., Ollero, A., 2011. Experimental results in multi-UAV coordination for disaster management and civil security applications. Journal of intelligent \& robotic systems, 61(1-4), 563-585.

Pajares, G., 2015. Overview and Current Status of Remote Sensing Applications Based on Unmanned Aerial Vehicles (UAVs). Photogrammetric Engineering \& Remote Sensing, 81(4), 281 - 329.

Pepe, M., Fregonese, L., Scaioni, M., 2018. Planning airborne photogrammetry and remote-sensing missions with modern platforms and sensors. European Journal of Remote Sensing, 51(1), 412-436.

Piras, M., Di Pietra, V., Visintini, D., 2017. 3D modeling of industrial heritage building using COTSs system: Test, limits and performances. $42,281-288$.

Pozyx Labs, 2015. Pozyx positioning system. https://www.pozyx.io/.

Remondino, F., Barazzetti, L., Nex, F., Scaioni, M., Sarazzi, D., 2011. UAV photogrammetry for mapping and 3D modelingcurrent status and future perspectives. ISPRS - International Archives of the Photogrammetry, Remote Sensing and Spatial Information Sciences, 38(1), C22.

Schaback, R., 1985. Convergence analysis of the general Gauss-Newton algorithm. Numerische Mathematik, 46(2), 281-309.

Tiemann, J., Schweikowski, F., Wietfeld, C., 2015. Design of an UWB indoor-positioning system for UAV navigation in GNSSdenied environments. Indoor Positioning and Indoor Navigation (IPIN), 2015 International Conference on, IEEE, 1-7. 
Zahran, S., Mostafa, M., Masiero, A., Moussa, A., Vettore, A., El-Sheimy, N., 2018. Micro-RADAR and UWB aided UAV navigation in GNSS denied environment. ISPRS - International Archives of the Photogrammetry, Remote Sensing and Spatial Information Sciences, XLII-1, 469-476.

Zhang, C., Kovacs, J. M., 2012. The application of small unmanned aerial systems for precision agriculture: a review. Precision agriculture, 13(6), 693-712. 